\section{Abstractions}

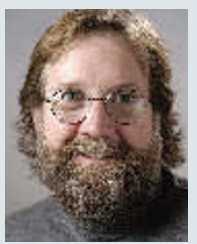

\section{LAST AUTHOR}

Genetic mutation is central to evolution, but mutations that improve one aspect of a protein's function can compromise another. Until recently, gene duplication was thought to lead to the development of genes with novel functions, as one copy of the gene would be free to evolve while the other performed its original function. But this, it turns out, rarely happens - more often, an original gene's functions are simply split between the two copies. Sean Carroll, at the University of WisconsinMadison, and his student Chris Hittinger devised a series of assays to trace how two genes - GAL1 and GAL3 - in the yeast Saccharomyces cerevisiae evolved from a single gene after duplication in a distant ancestor (see page 677). They suggest that genes that evolve in this way do so to overcome constraints present in the original gene.

Most of your work has been with fruitflies. Why do this work in yeast?

To find out how duplicate genes had changed, we needed to be able to measure very small differences in organismal fitness. We didn't think this would be possible in fruitflies - we needed the greater power of billions of offspring quickly, and yeast provided this.

Was it surprising that most of the two genes' differences were regulatory in nature? Yes. The protein Gal1 is galactokinase - an enzyme - and Gal3 acts to regulate its transcription. The original protein would have performed both these functions. We thought the divergence of these proteins was the main story, but the data told us otherwise. Gal1 still has a lot of regulatory activity, and Gal3 only recently lost the last of its enzymatic activity.

This duplication is thought to have occurred 100 million years ago - why did it take so long for enzymatic activity to be lost? I can think of many examples of genes deteriorating relatively quickly. But in this case, Gal3 still has to bind galactose in its regulatory role, so perhaps that constraint limited the loss of enzymatic activity.

Besides genetic duplication and divergence, might there be other evolutionary 'tools'? There are other mutational pathways, so yes. For instance, Chris showed that one way to make a better regulatory element is to delete bases so that transcription-factor binding sites get closer together.

Why did you write The Making of the Fittest, an evolution book for the general public? The pace of discovery in evolutionary science has really quickened. The quality of evidence and the clarity of how evolution works are now easier to talk about. And, happily, people want to know about evolution.

\section{MAKING THE PAPER}

\author{
Carolyn Porco
}

\section{Spacecraft's images suggest one of Saturn's moons may host water.}

The Cassini spacecraft took seven years to reach Saturn. But for Carolyn Porco, who leads the Cassini imaging team at the Space Science Institute (SSI) in Boulder, Colorado, the images it sent back were well worth the wait. Most exciting of all was the revelation that one of the planet's moons may have the essential ingredients to support life.

A veteran of the 1980s Voyager space mission, Porco was well aware that the outer Solar System is not the barren wasteland it was once thought to be. Images from Voyager, for example, had shown some of Jupiter's and Neptune's moons to be geologically active. But this knowledge didn't dampen the thrill of Cassini's discovery that Enceladus, one of Saturn's 60 moons, spews jets of vapour containing organic material and tiny, icy particles from its south pole. This spectacular finding demonstrates present-day geological activity on a small, cold moon.

Enceladus is only about 500 kilometres in diameter, but the jets can shoot thousands of kilometres into space. The particles they contain eventually make their way into Saturn's E ring the diffuse outermost ring, which is composed of microscopic icy particles. Long before Cassini arrived at Saturn, scientists had suspected Enceladus to be the E ring's source of material, but they never expected this dramatic phenomenon. And the drama matches the implications: "It's not out of the question that these jets are the result of liquid water," says Porco.

Infrared measurements by Cassini showed south pole 'hot spots' almost 100 kelvin warmer than the surface temperature expected from thermal equilibrium with sunlight. The hot spots are associated with four distinctive linear cracks - dubbed 'tiger-stripe' fractures - in the moon's surface. "We were in a tizzy," says Porco, "because, if we could confirm the presence of

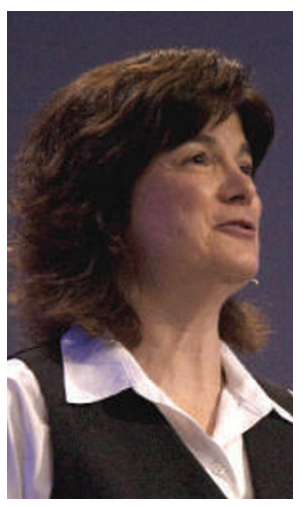

water in addition to organic materials and warmer temperatures, we may have stumbled upon a habitable zone in our Solar System. This is an explorer's dream come true."

The next step was to determine the locales of the jets. Porco asked SSI planetary scientist Joseph Spitale to triangulate the surface locations of each jet. To ensure that the measurements were made without prejudice, she didn't tell him her hypothesis of an association between the hot spots and the jets.

Spitale's measurements "hit the jackpot", Porco says - all of the prominent jets emerge from one of the four tiger-stripe fractures, and most coincide with one of the hot spots, confirming a causal relationship between the south pole's anomalous heat and jet activity (see page 695).

Geysering activity powered by pressurized liquid water trapped beneath the south pole is a possible mechanism for the jets; another is water vapour evaporating from warm ice. Unfortunately, no single observation from Cassini can answer this question. Although it will take several lines of evidence, Porco says that the prospect of finding an extraterrestrial habitat suitable for life is the greatest thrill any scientist could hope to experience.

Cassini's mission extends until 2010. A handful of additional Enceladus flybys are planned, and Porco and Spitale predict that other hot spots will be found in the fracture area. Planning future observations while analysing incoming data is all-consuming, says Porco, adding that "Cassini hasn't been a mission so much as a way of life". That lifestyle looks set to continue: Porco already thinks Enceladus merits another mission. "In my mind, it's the go-to place for investigating issues of astrobiological interest," she says.

\title{
FROM THE BLOGOSPHERE
}

On Nature's social networking website, Nature Network, we've created a new group called 'Ask the Nature Editor' (http:// network.nature.com/group/ askthenatureeditor). Here, several editors have agreed to answer your burning questions about publishing in Nature (and the other Nature journals), peer review and the scientific publishing process. We invite you to join the group - it's your chance to learn more about what goes on here, straight from the editors themselves. In the coming months, we'll have other Q\&A rounds focused on different publishing topics, such as careers in scientific publishing and online communications tools.

Current topics of discussion in the Q\&A forum (http:// network.nature.com/forum/ askthenatureeditor) include training for peer reviewers; what happens when manuscripts are submitted that are not written in journal style or not well written; and the pros and cons of submitting modified famous works of art as suggestions for the journal's cover.

If there are any topics you'd like the forum to cover in future, please post your suggestions at the URL above. We look forward to seeing you there. 